\title{
Infant orofacial movements: Inputs, if not outputs, of early imitative ability?
}

Commentary on Keven \& Akins (2017). Neonatal imitation in context:

Sensorimotor development in the perinatal period. Behavioral and Brain

Sciences.

Eoin P. O'Sullivan ${ }^{a}$ and Christine A. Caldwell ${ }^{b}$

${ }^{a}$ Faculty of Natural Sciences, Behaviour and Evolution Research Group, The University of Stirling, Stirling, UK, FK9 4LA.

\section{eoin.osullivan@stir.ac.uk}

\section{c.a.caldwell@stir.ac.uk}

\section{http://rms.stir.ac.uk/converis-stirling/person/10922}

\section{https://sites.google.com/site/christineannacaldwell/}

\begin{abstract}
According to Keven \& Akins (K\&A), infant orofacial gestures may not reflect imitative responses. Here we emphasise that these actions nonetheless represent a significant feature of the infant's early sensorimotor experience, and therefore may play a key role in the development of imitative capacities. We discuss how the ideas proposed in the target article could contribute substantially to experiential accounts of imitation.
\end{abstract}

This article has been accepted for publication in Behavioral and Brain Sciences. This version is free to view and download for private research and study only. Not for re-distribution, re-sale or use in derivative works. (C) 2017 Cambridge University Press 
Keven \& Akins (K\&A) have contributed an important new perspective to a growing body of literature that has challenged longstanding assumptions regarding the existence of innate imitative ability in infants (Jones 1996; Oostenbroek et al. 2016; Ray \& Heyes 2011). We agree with their assertion that infant orofacial "gestures" likely do not in fact arise as a consequence of imitation of others' actions. Nonetheless, evidence of imitation in slightly older children is indisputable, and rejection of a nativist interpretation necessitates adequate alternative explanation(s) of such proficiency. We believe there is now a pressing need for consideration and investigation of such alterative accounts. In this commentary we would specifically like to advocate the importance of understanding how early experience is involved in the development of imitative ability.

Early work by Piaget (1962) suggested imitation developed in a step-wise manner over the first two years, a finding now supported by more systematic research (Jones 2007). Some theoretical models have proposed that this developmental trend is scaffolded by sensorimotor experience; for example, when an infant observes their own actions, or when a caregiver's actions correspond to those of the infant. According to this view, this experience builds associations between sensory and motor representations of the same action that will later facilitate imitation (Brass \& Heyes 2005; Ray \& Heyes 2011). It follows from these assumptions that the stereotypic actions discussed by K\&A are likely to play a significant role in the development of these associations. Indeed, evidence suggests that caregivers frequently imitate infant action (Flynn et al. 2004; Pawlby 1977), and stereotypies may increase during caregiver interaction (Thelen 1981). Opportunities for caregiver imitation may therefore largely consist of rhythmic stereotypies. These 
imitative interactions could provide the infant with rich sensorimotor experience critical to the formation of associations which eventually underpin their own imitative capacities. Therefore, orofacial stereotypies in infancy might more accurately be viewed as inputs into, rather than outputs of, early imitation.

Our own work on action imitation in older children has found that the likely extent of sensorimotor experience of synchronous activity appears to be related to the automaticity of imitative responses (O'Sullivan et al. under review). Although such effects have yet to be identified in infants, we would expect that nascent imitative abilities would be similarly affected by the aggregated experience of correspondences between sensory and proprioceptive feedback associated with particular actions. To conclusively demonstrate that sensorimotor experience is a key component of imitative development, researchers must document naturally occurring sensorimotor experience and its implications for imitation throughout the first year. The study of behavioural stereotypies offers valuable groundwork that could inform the approach taken within such an enterprise.

A systematic developmental approach to the study of infant stereotypies and their environmental triggers could also considerably strengthen K\&A's argument, as well as augmenting our understanding of the development of imitation. We are sympathetic towards the notion that apparently imitative responses in neonates may in fact be an artefact of stereotypic behaviour triggered by arousing stimuli. Thelen's $(1979 ; 1981)$ observational studies of rhythmic stereotypies in infancy (cited in the target article) have 
provided important groundwork in this respect, identifying a wide range of stereotypies and some apparent releasers. However, to further elucidate the reasons for apparently imitative correspondences during infancy, experimental approaches (similar to those used in neonatal imitation studies) could be used to systematically investigate the responses elicited by a comprehensive suite of actions modelled by a caregiver (including, importantly, those identified as common rhythmic stereotypies with different peak postnatal frequencies). Arousal theories propose that correspondences between the actions of model and infant are found in studies of neonatal imitation because the model's actions tend to be arousing, and the infant's actions are developmental stereotypies which would increase in response to any arousing stimulus. If this is the case, it should follow that any stimulus known to release a particular stereotypy during a specific postnatal phase of development, should also trigger the production of other stereotypies during other phases of development (i.e. in accordance with their documented peak production frequencies). Such evidence would corroborate the conclusion that correspondences are coincidental, rather than imitative responses. Nonetheless, assuming a role for experience in the development of imitation, one might also expect that over the course of development the specific actions produced by the infant should show an increasing tendency to match those of the model, across the full range of behaviours (and taking into account expected production frequencies).

Finally, we would also like to highlight the implications of the argument in the target article, along with an experiential account of imitative ability, for understanding the social role of imitation. A rich literature has explored how imitation in children and 
adults is associated with affiliation toward imitators (Chartrand \& Bargh 1999; Over et al. 2013). It has been suggested, likely because of assumptions about the innateness of imitative ability, that this is an adaptive response which has been subject to evolutionary selection pressure (Lakin \& Jefferis 2003). However, K\&A have highlighted how behavioural stereotypies are linked with arousal in infants (Jones 1996; Nagy \& Molnar 2004) and, as proposed above, these stereotypies may also provide opportunities for imitative interactions. Therefore, such interactions may further associate the experience of imitative correspondences within a prosocial context of positive arousal. To our knowledge, no research has yet examined how imitation becomes such a pervasively social phenomenon in childhood. However, as strong nativist views of imitative ability continue to be questioned, we must explore how an infant's social environment might facilitate the development of links between imitation and other experiences, including physiological and psychological states.

We hope that our reflections will encourage others to take an interest in the role of learning in the formation of flexible adaptive social behaviours, and to further consider how such processes are influenced by universal developmental phenomena such as behavioural stereotypies.

Brass, M. \& Heyes, C. (2005) Imitation: Is cognitive neuroscience solving the correspondence problem? Trends in cognitive sciences 9(10):489-95. 
Chartrand, T. L. \& Bargh, J. A. (1999) The chameleon effect: The perception-behaviour link and social interaction. Journal of Personality and Social Psychology 76(6):893-910.

Flynn, V., Masur, E. F. \& Eichorst, D. L. (2004) Opportunity versus disposition as predictors of infants' and mothers' verbal and action imitation. Infant Behavior and Development 27(3):303-14.

Jones, S. S. (1996) Imitation or exploration? Young infants' matching of adults' oral gestures. Child Development 67(5):1952-69.

Jones, S. S. (2007) Imitation in infancy: The development of mimicry. Psychological Science 18(7):593-9.

Lakin, J. \& Jefferis, V. (2003) The chameleon effect as social glue: Evidence for the evolutionary significance of nonconscious mimicry. Journal of Nonverbal Behaviour 27:145-62.

Nagy, E. \& Molnar, P. (2004) Homo imitants or homo provocans? Human imprinting model of neonatal imitations. Infant Behavior and Development 27(1):54-63.

Oostenbroek, J., Suddendorf, T., Nielsen, M., Redshaw, J., Kennedy-Costantini, S., Davis, J., Clark, S. \& Slaughter, V. (2016) Comprehensive longitudinal study challenges the existence of neonatal imitation in humans. Current Biology 26(10): 1334-38. Elsevier.

Over, H., Carpenter, M., Spears, R. \& Gattis, M. (2013) Children selectively trust individuals who have imitated them. Social Development 22(2):215-24.

O’Sullivan, E. P., Bijvoet-van den Berg, S. \& Caldwell, C. A. (under review) Automatic imitation effects are influenced by experience of synchronous action in children. 
Pawlby, S. (1977) Imitative interaction. In: Studies in mother-infant interaction, ed. H. Schaffer, pp. 203-33. Academic.

Piaget, J. (1962) Play, dreams, and imitation in childhood. Norton.

Ray, E. \& Heyes, C. (2011) Imitation in infancy: The wealth of the stimulus. Developmental Science 14(1):92-105.

Thelen, E. (1979) Rythmical stereotypies in normal human infants. Animal Behavior 27(1952):699-715.

Thelen, E. (1981) Kicking, rocking, and waving: Contextual analysis of rhythmical stereotypies in normal human infants. Animal Behaviour 29(1):3-11. 